\title{
Acupuncture decreased the risk of coronary heart disease in patients with fibromyalgia in Taiwan: a nationwide matched cohort study
}

Mei-Yao Wu', Ming-Cheng Huang ${ }^{2,3}$, Jen-Huai Chiang ${ }^{4,5}$, Mao-Feng Sun ${ }^{2,3}$, Yu-Chen Lee $2,6^{*+}$ and Hung-Rong Yen ${ }^{1,2,3,5^{*+}}$ (i)

\begin{abstract}
Background: The aim of this study was to understand whether acupuncture can decrease the risk of coronary heart disease (CHD) in patients with fibromyalgia.

Methods: Using data from the Taiwanese National Health Insurance Research Database, we performed a propensity score-matched cohort study to analyze patients with fibromyalgia diagnosed between 1 January 2000 and 31 December 2010. Patients who received acupuncture treatment, beginning with their initial date of fibromyalgia diagnosis and extending to 31 December 2010, were regarded as the acupuncture cohort. The no-acupuncture cohort comprised patients who never received acupuncture through 31 December 2010. A Cox regression model was used to adjust for age, sex, comorbidities, and drugs used. The HRs of the acupuncture and no-acupuncture cohorts were compared.

Results: After performing a 1:1 propensity score match, 58,899 patients in both cohorts were identified. Baseline characteristics were similar in both cohorts. The cumulative incidence of CHD was significantly lower in the acupuncture cohort (log-rank test, $p<0.001$ ). In the follow-up period, 4389 patients in the acupuncture cohort (17. 44 per 1000 person-years) and 8133 patients in the no-acupuncture cohort (38.36 per 1000 person-years) developed CHD (adjusted HR 0.43, 95\% Cl 0.41-0.45). The beneficial effect of acupuncture on the incidence of CHD was independent of age, sex, comorbidities, and statins used.

Conclusions: Our study confirmed that acupuncture reduced the risk of CHD in patients with fibromyalgia in Taiwan. Further clinical and mechanistic studies are warranted.
\end{abstract}

Keywords: Acupuncture, Coronary heart disease, Fibromyalgia, National Health Insurance Research Database, Taiwan

\section{Background}

Fibromyalgia, characterized by chronic widespread pain, commonly presents with associated symptoms such as fatigue, sleep disturbance, depression, cognitive dysfunction, and headache $[1,2]$. The American College of

\footnotetext{
*Correspondence: d5167@mail.cmuh.org.tw; hungrongyen@gmail.com ${ }^{\dagger}$ Equal contributors

2Department of Chinese Medicine, China Medical University Hospital, 2 Yude Road, North District, Taichung, Taiwan

${ }^{1}$ Research Center for Traditional Chinese Medicine, Department of Medical Research, China Medical University Hospital, 2 Yude Road, North District, Taichung, Taiwan

Full list of author information is available at the end of the article
}

Rheumatology published the criteria for fibromyalgia, which were modified in 2010 [3]. The global mean prevalence of fibromyalgia in the general population was $2.7 \%$, and the female-to-male ratio was 3:1 [4]. The quality of life (QOL) of patients with fibromyalgia is always impaired, and about one-third of patients have difficulties in performing the activities of daily living. The high incidence of comorbidities is another health care problem. Researchers in previous studies reported that patients with fibromyalgia in the United States were two to seven times more likely to have comorbidities [5]. According to the U.S. National Health Interview Survey, 
myocardial infarction occurred more than twice as often in patients with fibromyalgia as in patients without fibromyalgia [6]. In Korea, chronic emotional stress in postmenopausal women with fibromyalgia impaired myocardial function [7]. In Taiwan, a population-based cohort study revealed that the risk of coronary heart disease (CHD) in patients with fibromyalgia was $47 \%$ higher than in the general population [8].

Current treatment strategies for fibromyalgia include pharmacological and nonpharmacological methods. Pregabalin, duloxetine, and milnacipran are U.S. Food and Drug Administration (FDA)-approved drugs for treating patients with fibromyalgia, but the efficacy of these drugs has been questioned because of the small sample sizes in clinical trials [9]. Nonpharmacological treatments of fibromyalgia include acupuncture, massage, and exercise. Because the pathological mechanisms of fibromyalgia are not yet fully understood, current treatments are focused on attenuating symptoms and improving QOL.

Animal studies and clinical trials revealed that acupuncture has a beneficial effect in the treatment of fibromyalgia $[10,11]$. However, there is no long-term follow-up study showing whether acupuncture can decrease the risk of $\mathrm{CHD}$ in patients with fibromyalgia. The Taiwanese National Health Insurance Research Database (NHIRD) provides information on the entire population of Taiwan with long-term follow-up data for interventions. The advantage of using this database is that it prevents sampling bias [12]. The National Health Insurance (NHI) program was implemented in 1995 in Taiwan by the National Health Insurance Administration (NHIA). Traditional Chinese medicine (TCM) services have been reimbursed through the NHI program since 1996 [13]. The use of TCM services, including herbal medicine and acupuncture, is common in Taiwan for the treatment of many kinds of disease [14-17]. To understand whether acupuncture can decrease the risk of CHD in patients with fibromyalgia, we investigated a randomly selected sample of 1 million people enrolled in the NHIRD from 2000 to 2010 .

\section{Methods}

\section{Data sources}

We conducted a nationwide, population-based, 1:1 propensity score-matched cohort study by analyzing data derived from the NHIRD. The data source of our study was the Longitudinal Health Insurance Database 2000, which contains all the original claims data of 1 million beneficiaries randomly sampled from the registry of all beneficiaries in 2000. The sampled patients exhibited no significant difference in age, sex, birth year, or average insured payroll-related costs from the general population. The International Classification of Diseases, Ninth Revision, Clinical Modification (ICD-9-CM), codes were used for diagnoses. Because the NHIRD contains identified secondary data for research, the present study was waived from the need for informed consent. This study was approved by the research ethics committee of China Medical University and Hospital (CMUH104-REC2-115).

\section{Study cohort identification}

Patients newly diagnosed with fibromyalgia from 1 January 2000 to 31 December 2010 were identified (Fig. 1). To be included, patients with fibromyalgia should have had at least two ambulatory or inpatient claims with the diagnosis of ICD-9-CM codes 729.0 and 729.1. The exclusion criteria included age younger than 18 years, incomplete information on age and sex, and withdrawal from the NHIRD during the follow-up period. Patients who received acupuncture from their initial diagnosis of fibromyalgia to 31 December 2010 were identified as the acupuncture cohort. Propensity score approaches were used to reduce confounding by the indication of acupuncture treatment. We used a 1:1 propensity score match by sex, age (per 5 years), comorbidities, drugs used, and the year in which fibromyalgia was diagnosed through multiple logistic regression analysis. Ultimately, equal numbers of patients in the acupuncture and no-acupuncture cohorts were analyzed in this study. The immortal time of acupuncture cohort was the period between the first time of receiving acupuncture and the date of initial diagnosis with fibromyalgia. The claims data for both cohorts were assessed from the initial diagnosis date to 31 December 2011 (the end of this study).

\section{Covariate assessment}

Sociodemographic factors included age and sex. Patients were divided into three groups according to their age (18-39 years, 40-59 years, and older than 60 years). Baseline comorbidities were considered present if ICD9-CM codes appeared two or more times in outpatient or inpatient claims before the initial diagnosis of fibromyalgia, which included diabetes mellitus (DM; ICD-9CM code 250), hypertension (ICD-9-CM codes 401405), hyperlipidemia (ICD-9-CM code 272), congestive heart failure (ICD-9-CM codes 402.01, 402.11, 402.91, $404.01,404.03,404.11,404.13,404.91,404.93$, and 428.0), stroke (ICD-9-CM codes 430-438), depression (ICD-9-CM codes 296.2-296.3, 300.4, and 311), anxiety (ICD-9-CM codes 300.0, 300.2, 300.3, 308.3, and 308.91), alcoholism or alcohol-related disorders (ICD-9CM codes 291, 303, 305.00-305.03, 790.3, and V11.3), tobacco dependence (ICD-9-CM code 305.1), and obesity (ICD-9-CM codes 278 and A183). 


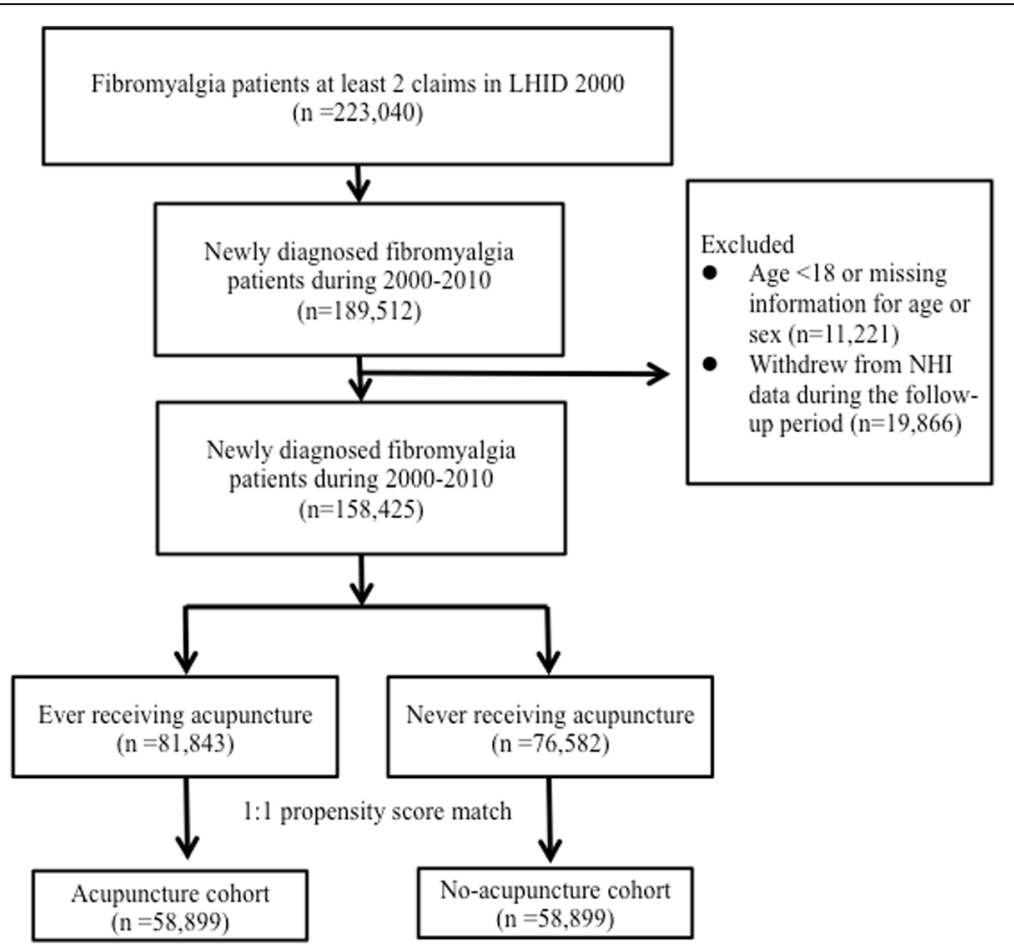

Fig. 1 Study population flowchart. After the exclusion process, we identified 158,425 patients with fibromyalgia newly diagnosed between 2000 and 2010. After matching 1:1 by sex, age, comorbidities, and drugs used, the acupuncture cohort and no-acupuncture cohorts each contained 58,899 patients. LHID 2000 Longitudinal Health Insurance Database 2000, NHI National Health Insurance

\section{Types of acupuncture and disease categories in the acupuncture cohort}

We analyzed the acupuncture type that patients received by the treatment codes, which included manual acupuncture of TCM type (B41, B42, B45, B46, B80-B84, B90-B94, P27041, P31103, P32103, and P33031) and electroacupuncture (B43, B44, B86-B89, and P33032). Disease categories for which patients with fibromyalgia received acupuncture were identified by the ICD-9-CM codes. More than one ICD-9-CM code may be recorded when patients receive acupuncture, so the total numbers of patients in all categories were more than the number of patients in the acupuncture cohort.

\section{Statistical analyses}

We compared the baseline characteristics of the acupuncture and no-acupuncture cohorts using standardized mean differences. Standardized mean differences with less than $0.1 \mathrm{SD}$ indicated a negligible difference in mean values or proportions between the two cohorts. The HR and 95\% CI were calculated for each variable by Cox proportional hazards regression. The difference between the two cohorts in the development of CHD was estimated using the Kaplan-Meier method and the logrank test.

Statistical analysis was performed and figures were created using SAS 9.4 (SAS Institute, Cary, NC, USA) and $\mathrm{R}$ software ( $\mathrm{R}$ Foundation for Statistical Computing, Vienna, Austria). $p<0.05$ in two-tailed tests indicated statistical significance.

\section{Results}

Using the NHIRD, we identified 189,512 patients with fibromyalgia newly diagnosed between 1 January 2000 and 31 December 2010 (Fig. 1). After excluding patients with missing information or lacking follow-up, 158,425 patients were included. Among included patients, 81,843 received acupuncture and 76,582 patients never received acupuncture from the initial fibromyalgia diagnosis date to 31 December 2010. To minimize the differences in basic data between these two cohorts, we used a 1:1 propensity score match to randomly select 58,899 patients each for the acupuncture and no-acupuncture cohorts.

The baseline characteristics of both cohorts are shown in Table 1, and they were similar in sex, age, comorbidities, and drugs used. The proportion of women was higher than men in both cohorts, and the dominant age group was 40-59 years. The most common comorbidity was hypertension, which was present in more than $20 \%$ of patients. Among the patients with fibromyalgia, 17\% had hyperlipidemia, 11\% had anxiety, $10.6 \%$ had DM, 6.9\% had stroke, $4.9 \%$ had depression, and $1 \%$ had congestive heart failure. 
Table 1 Characteristics of patients with fibromyalgia in the present study

\begin{tabular}{|c|c|c|c|}
\hline \multirow[t]{2}{*}{ Variable } & $\begin{array}{l}\text { No } \\
\text { acupuncture } \\
(n=58,899)\end{array}$ & $\begin{array}{l}\text { Acupuncture } \\
(n=58,899)\end{array}$ & \multirow[t]{2}{*}{$\begin{array}{l}\text { Standardized } \\
\text { mean difference }\end{array}$} \\
\hline & $\%$ & $\%$ & \\
\hline
\end{tabular}

\begin{tabular}{|c|c|c|c|c|c|}
\hline \multicolumn{6}{|l|}{ Sex } \\
\hline Female & 33,331 & 56.59 & 33,447 & 56.79 & 0.00 \\
\hline Male & 25,568 & 43.41 & 25,452 & 43.21 & \\
\hline \multicolumn{6}{|l|}{ Age group, years } \\
\hline 18-39 & 24,487 & 41.57 & 23,572 & 40.02 & 0.03 \\
\hline $40-59$ & 27,481 & 46.66 & 29,748 & 50.51 & 0.077 \\
\hline$\geq 60$ & 6931 & 11.77 & 5579 & 9.47 & 0.07 \\
\hline Mean $( \pm S D)$, years & \multicolumn{2}{|c|}{$44.57(15.43)$} & \multicolumn{2}{|c|}{$44.28(14.44)$} & 0.02 \\
\hline \multicolumn{6}{|l|}{ Baseline comorbidities } \\
\hline Diabetes mellitus & 6252 & 10.61 & 6098 & 10.35 & 0.009 \\
\hline Hypertension & 12,457 & 21.15 & 12,036 & 20.43 & 0.018 \\
\hline Hyperlipidemia & 10,097 & 17.14 & 10,034 & 17.04 & 0.00 \\
\hline $\begin{array}{l}\text { Congestive heart } \\
\text { failure }\end{array}$ & 569 & 0.97 & 508 & 0.86 & 0.01 \\
\hline $\begin{array}{l}\text { Cerebrovascular } \\
\text { diseases }\end{array}$ & 4044 & 6.87 & 3786 & 6.43 & 0.01 \\
\hline Depression & 2863 & 4.86 & 2840 & 4.82 & 0.002 \\
\hline Anxiety & 6551 & 11.12 & 6600 & 11.21 & 0.003 \\
\hline $\begin{array}{l}\text { Alcoholism or } \\
\text { alcohol-related } \\
\text { disorders }\end{array}$ & 311 & 0.53 & 316 & 0.54 & 0.00 \\
\hline Tobacco dependence & 289 & 0.49 & 270 & 0.46 & 0.005 \\
\hline Obesity & 439 & 0.75 & 427 & 0.72 & 0.00 \\
\hline \multicolumn{6}{|l|}{ Drugs used } \\
\hline Oral steroids & 39466 & 67.01 & 39495 & 67.06 & 0.001 \\
\hline NSAIDs & 57613 & 97.82 & 57734 & 98.02 & 0.014 \\
\hline Statins & 7971 & 13.53 & 7937 & 13.48 & \\
\hline
\end{tabular}

Types of acupuncture

Manual acupuncture of TCM type

$\begin{array}{llll}\begin{array}{l}\text { Electroacupuncture } \\ \text { Combination of }\end{array} & - & 2142 & 3.64 \\ \begin{array}{l}\text { manual acupuncture } \\ \text { and electroacupuncture }\end{array} & 6287 & 10.67 \\ \begin{array}{l}\text { Duration between the } \\ \text { initial diagnosis to the }\end{array} & & \\ \text { first acupuncture } \\ \text { treatment, days, mean } \\ \text { (median) } \\ \begin{array}{l}\text { Acupuncture visits, } \\ \text { mean }\end{array} \\ \begin{array}{l}\text { Follow-up duration, } \\ \text { years, mean (median) }\end{array} & - & \\ \end{array}$
years, mean (median)
The proportions of patients diagnosed with alcoholism or alcohol-related disorders, tobacco dependence, and obesity were similar in both cohorts. Almost all patients in both cohorts used nonsteroidal antiinflammatory drugs (NSAIDs), and approximately 67\% of patients used oral steroids. About $13.5 \%$ of patients took statins for hyperlipidemia in the no-acupuncture and acupuncture cohorts. The mean duration between the initial diagnosis of fibromyalgia and the first time receiving acupuncture was approximately 919 days. The average number of acupuncture visits was 7.45. Most patients (85\%) received manual acupuncture of TCM type, and $3.6 \%$ patients received electroacupuncture. About $10.7 \%$ of patients received both types of treatment.

During the follow-up period, there were 12,522 patients included in our study who developed CHD (Table 2). The incidence of CHD in patients with fibromyalgia increased depending on age (adjusted HRs 4.24 and 6.24 in 40-59 years and $\geq 60$ years age groups, respectively). Patients with fibromyalgia with comorbidities also developed CHD more easily than patients without comorbidities. Overall, the incidence of CHD was significantly lower in the acupuncture cohort than in the no-acupuncture cohort (adjusted HR 0.43, 95\% CI 0.41-0.45).

Figure 2 reveals that the cumulative incidence of CHD was significantly lower in the acupuncture cohort during the follow-up period (log-rank test, $p<0.001$ ). In this study, 4389 patients in the acupuncture cohort (17.44 per 1000 person-years) and 8133 patients in the noacupuncture cohort (38.36 per 1000 person-years) developed CHD (adjusted HR 0.43, 95\% CI 0.41-0.45) (Table 3). The beneficial effect of acupuncture on the incidence of CHD was observed in both female and male patients (adjusted HR 0.48 in women, 95\% CI 0.46-0.51; adjusted HR 0.47 in men, 95\% CI 0.45-0.50). Although the risk of CHD gradually increased with age, acupuncture significantly decreased the incidence of CHD in all age groups. Acupuncture decreased the risk of CHD in patients with fibromyalgia with or without comorbidities. Regardless of whether patients took oral steroids, NSAIDs, or statins, fewer patients in the acupuncture cohort developed CHD than in the no-acupuncture cohort.

Table 4 reveals the top ten disease categories for which the patients with fibromyalgia in the acupuncture cohort received acupuncture treatment. The most common conditions leading to visiting acupuncture doctors were disorders of the musculoskeletal system and connective tissues that are highly related to fibromyalgia. Injury was also a common reason for receiving acupuncture.

\section{Discussion}

To our knowledge, our present nationwide populationbased study is the first to reveal that acupuncture 
Table 2 Hazard ratios of coronary heart disease associated with acupuncture and covariates among patients with fibromyalgia

\begin{tabular}{|c|c|c|c|c|c|c|c|}
\hline Variable & Number of events $(n=12,522)$ & Crude HR & $(95 \% \mathrm{Cl})$ & $p$ Value & Adjusted HR & $(95 \% \mathrm{Cl})$ & $p$ Value \\
\hline \multicolumn{8}{|l|}{ Received acupuncture } \\
\hline No & 8133 & 1.00 & Reference & & 1.00 & Reference & \\
\hline Yes & 4389 & 0.48 & $(0.46-0.5)$ & $<0.0001$ & 0.43 & $(0.41-0.45)$ & $<0.0001$ \\
\hline \multicolumn{8}{|l|}{ Sex } \\
\hline Female & 7138 & 1.00 & Reference & & 1.00 & Reference & \\
\hline Male & 5384 & 0.98 & $(0.95-1.02)$ & 0.3907 & 1.03 & $(0.99-1.07)$ & 0.1072 \\
\hline \multicolumn{8}{|l|}{ Age group, years } \\
\hline 18-39 & 1248 & 1.00 & Reference & & 1.00 & Reference & \\
\hline $40-59$ & 7946 & 5.24 & $(4.94-5.56)$ & $<0.0001$ & 4.24 & $(3.99-4.51)$ & $<0.0001$ \\
\hline$\geq 60$ & 3328 & 11.33 & $(10.62-12.1)$ & $<0.0001$ & 6.24 & $(5.81-6.71)$ & $<0.0001$ \\
\hline \multicolumn{8}{|c|}{ Baseline comorbidities (reference $=$ nonsite comorbidity) } \\
\hline Diabetes mellitus & 2994 & 2.9 & $(2.78-3.02)$ & $<0.0001$ & 1.37 & $(1.31-1.44)$ & $<0.0001$ \\
\hline Hypertension & 6188 & 4.03 & $(3.89-4.18)$ & $<0.0001$ & 2.20 & $(2.11-2.3)$ & $<0.0001$ \\
\hline Hyperlipidemia & 4452 & 2.88 & $(2.78-2.99)$ & $<0.0001$ & 1.63 & $(1.56-1.7)$ & $<0.0001$ \\
\hline Congestive heart failure & 298 & 3.19 & $(2.85-3.58)$ & $<0.0001$ & 1.17 & $(1.04-1.32)$ & 0.0078 \\
\hline Cerebrovascular diseases & 1966 & 2.81 & $(2.68-2.95)$ & $<0.0001$ & 1.25 & $(1.19-1.32)$ & $<0.0001$ \\
\hline Depression & 803 & 1.48 & $(1.38-1.59)$ & $<0.0001$ & 1.1 & $(1.02-1.18)$ & 0.0131 \\
\hline Anxiety & 1960 & 1.61 & $(1.53-1.69)$ & $<0.0001$ & 1.21 & $(1.15-1.27)$ & $<0.0001$ \\
\hline Alcoholism or alcohol-related disorders & 57 & 0.97 & $(0.75-1.25)$ & 0.8031 & 0.93 & $(0.72-1.21)$ & 0.5941 \\
\hline Tobacco dependence & 39 & 0.9 & $(0.66-1.23)$ & 0.5098 & 0.83 & $(0.6-1.13)$ & 0.2391 \\
\hline Obesity & 109 & 1.34 & $(1.11-1.62)$ & 0.0024 & 1 & $(0.83-1.21)$ & 0.9629 \\
\hline \multicolumn{8}{|l|}{ Drugs used } \\
\hline Oral steroids & 7490 & 0.62 & $(0.6-0.64)$ & $<0.0001$ & 0.54 & $(0.52-0.56)$ & $<0.0001$ \\
\hline NSAIDs & 11,804 & 0.21 & $(0.19-0.23)$ & $<0.0001$ & 0.24 & $(0.22-0.26)$ & $<0.0001$ \\
\hline Statins & 2218 & 1.28 & $(1.22-1.34)$ & $<0.0001$ & 0.55 & $(0.52-0.58)$ & $<0.0001$ \\
\hline
\end{tabular}

NSAIDs Nonsteroidal anti-inflammatory drugs

${ }^{a}$ Crude HR represents relative hazard ratio

${ }^{b}$ Adjusted HR represents adjusted hazard ratio mutually adjusted for accepted acupuncture, age, sex, diabetes mellitus, hypertension, hyperlipidemia, congestive heart failure, cerebrovascular diseases, depression, anxiety, alcoholism or alcohol-related disorders, tobacco dependence, obesity, oral steroids, NSAIDs, and statins in Cox proportional hazards regression

decreases the risk of CHD in patients with fibromyalgia. As a popular treatment for fibromyalgia, acupuncture has been performed on patients with fibromyalgia in several clinical trials. However, the outcome evaluation was previously always focused on pain score, sleep quality, and QOL [10]. In our study, we found that the beneficial effects of acupuncture on developing CHD in patients with fibromyalgia were independent of sex, age, comorbidities, and anti-inflammatory drugs included in our data. Statins, the inhibitors of the 3-hydroxy-3methylglutaryl-coenzyme A reductase for treating hyperlipidemia, were previously reported to reduce the risk of cardiovascular diseases [18]. Our study revealed that regardless of whether patients with fibromyalgia took statins, acupuncture lowered the risk of CHD.

Our study revealed that more than half of patients with fibromyalgia in Taiwan have received acupuncture. A previous report revealed that the leading reason for patients in Taiwan to seek acupuncture treatment was diseases of the musculoskeletal system and connective tissue [19]. Fibromyalgia also belongs to this disease category. This is true not only in Taiwan, because patients with fibromyalgia also receive acupuncture in the other countries, such as Germany [20]. Previous studies in the United States, Spain, and Iran have demonstrated the efficacy of acupuncture in fibromyalgia [21-23].

A strength of our study is the comprehensive, largescale database that we used, Taiwan's NHIRD. This database provides an enormous sample size to reduce selection and participation bias, and it includes long-term follow-up data [12]. In addition, the characteristics of patients with fibromyalgia in our study were consistent with those in previous reports of female and middleaged predominance in other countries [6, 24]. These baseline characteristics were similar in both cohorts in our study. 


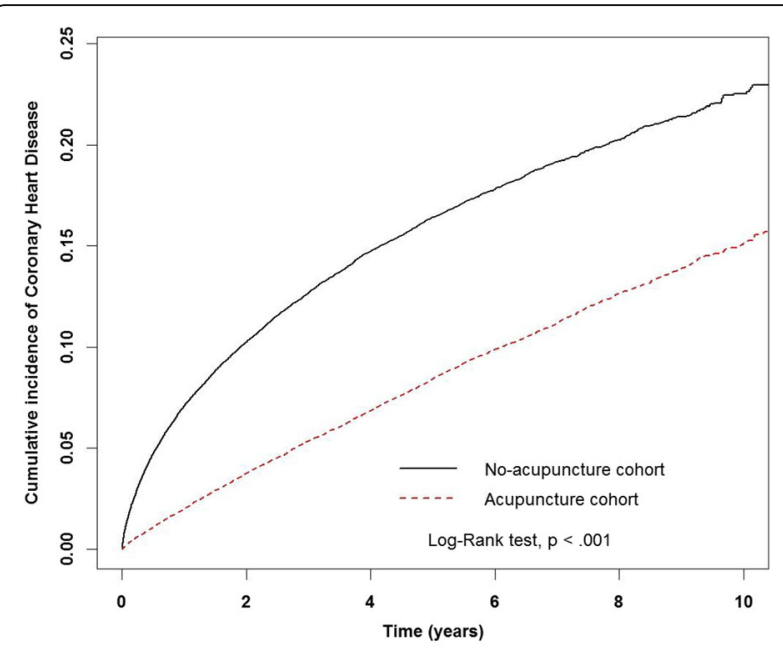

Fig. 2 Cumulative incidence of coronary heart disease (CHD) between the acupuncture cohort and the no-acupuncture cohort. The cumulative incidence of CHD in the acupuncture cohort (dashed line) is significantly lower than in the no-acupuncture cohort (solid line) (log-rank test, $p<0.001)$

In Taiwan, the majority of TCM doctors are graduated from baccalaureate TCM education programs (7- or 8year medical doctor programs) or postbaccalaureate TCM programs (5-year medical doctor programs) in universities. The graduated students must pass the board examination conducted by the government. The training programs on the manipulation of acupuncture are standardized. The duration of needle retention most commonly performed is about 20-30 minutes in Taiwan, and achievement of deqi is a basic requirement in acupuncture treatment.

Pregabalin was the first FDA-approved drug for treatment of fibromyalgia [1]. It was demonstrated to improve pain, sleep disturbance, and fatigue in adult patients with fibromyalgia [25]. In our present study, we did not compare the efficacy of acupuncture with pregabalin, because this drug was not reimbursed by Taiwanese NHIA for fibromyalgia until 2015. Whether pregabalin decreases the incidence of $\mathrm{CHD}$ in patients with fibromyalgia has never been reported. Furthermore, pregabalin has cardiac adverse effects because it may induce heart failure [26]. Acupuncture has been reported to attenuate both ischemic injury of the heart and heart failure [27, 28]. Whether acupuncture treatment provides benefits to those patients receiving pregabalin deserves future investigation.

Fibromyalgia is thought to arise from aberrant brain chemistry, function, and structure [29]. The effects of acupuncture have also been documented to affect the central nervous system to ameliorate fibromyalgia [30]. An increasing body of evidence suggests that central sensitization contributes to hyperalgesia and allodynia in patients with fibromyalgia. Activation of $N$-methyl-D-aspartate receptor (NMDAR) and cyclic adenosine monophosphate-responsive element binding protein promotes hyperalgesia [29]. In a previous study on fibromyalgia, researchers using a murine model found that activation of the dorsal root ganglion was suppressed by acupuncture [31]. Activation of cardiac NMDAR induces oxidative stress and facilitates atrial fibrillation that were attenuated by NMDAR antagonists [32,33]. Inhibition of NMDAR is the possible mechanism to prevent CHD in fibromyalgia, and whether acupuncture can suppress cardiac NMDAR in fibromyalgia needs further evaluation.

Other than the NMDAR pathway, the $5-\mathrm{HT}_{3}$ receptor may be another target of acupuncture to decrease CHD in fibromyalgia. The analgesic effect of acupuncture is also evident through the $5 \mathrm{HT}_{3}$ receptor in a rat model of collagenase-induced arthritis, and $5-\mathrm{HT}_{3}$ receptor antagonists have beneficial effects on autonomic cardiac dysfunction in patients with fibromyalgia [34, 35]. However, the results of our present study were contrary to those of a previous study involving transient receptor potential vanilloid 1 (TRPV1). Overexpression of TRPV1 in the spinal cord was suppressed by acupuncture in a fibromyalgia murine model [11], but TRPV1 in the endothelium prevents CHD [36]. The heart-protective effect of acupuncture in fibromyalgia may not be through the TRPV1 pathway.

Antidepression, anti-inflammation, and improvement of sleep quality are also possible mechanisms of acupuncture to prevent $\mathrm{CHD}$ in fibromyalgia. In previous reports, acupuncture improved sleep quality in patients with insomnia, which is highly associated with fibromyalgia and CHD [37-39]. Depression is a common associated symptom of fibromyalgia, and it increases the incidence of CHD through deregulation of the autonomic nervous system and the hypothalamic-pituitaryadrenal axis [40]. Acupuncture stabilized this axis, activated the hippocampal serotonin system, and suppressed inflammation to attenuate depression [41, 42].

Comorbidities of fibromyalgia, including DM, hypertension, and heart disease, are highly associated with chronic inflammation. Many previous studies of acupuncture were focused on the analgesic effect of acupuncture, but additional studies in recent years demonstrated that acupuncture attenuated inflammation. Acupuncture attenuated inflammation through the vagus nerve mediated by dopamine [43]. The combination of acupuncture and moxibustion relieved Crohn's disease by decreasing the ratio of Th17 to Treg cells in the intestinal mucosa [44]. These studies support that acupuncture has not only an analgesic effect but also an anti-inflammatory effect.

In addition, researchers in a previous study found that acupuncture before percutaneous coronary intervention in patients with acute myocardial infarction attenuated 
Table 3 Incidence rates and hazard ratios for coronary heart disease among patients with fibromyalgia with or without acupuncture treatment

\begin{tabular}{|c|c|c|c|c|c|c|c|c|}
\hline \multirow[t]{2}{*}{ Variable } & \multicolumn{3}{|c|}{ No acupuncture $(n=58,899)$} & \multicolumn{3}{|c|}{ Acupuncture $(n=58,899)$} & \multirow[b]{2}{*}{ Crude HR $(95 \% \mathrm{Cl})$} & \multirow[b]{2}{*}{ Adjusted HR $(95 \% \mathrm{Cl}$ ) } \\
\hline & Events & Person-years & $\mathbb{R}$ & Events & Person-years & IR & & \\
\hline Total & 8133 & 212,027 & 38.36 & 4389 & 251,711 & 17.44 & $0.48(0.46-0.50)^{b}$ & $0.43(0.41-0.45)^{\mathrm{b}}$ \\
\hline \multicolumn{9}{|l|}{ Sex } \\
\hline Female & 4650 & 120,934 & 38.45 & 2488 & 141,235 & 17.62 & $0.48(0.46-0.51)^{\mathrm{b}}$ & $0.43(0.41-0.45)^{b}$ \\
\hline Male & 3483 & 91,093 & 38.24 & 1901 & 110,476 & 17.21 & $0.47(0.45-0.5)^{b}$ & $0.45(0.43-0.48)^{b}$ \\
\hline \multicolumn{9}{|c|}{ Age group, years } \\
\hline $18-39$ & 877 & 92,941 & 9.44 & 371 & 94,477 & 3.93 & $0.42(0.38-0.48)^{b}$ & $0.39(0.35-0.44)^{b}$ \\
\hline $40-59$ & 5059 & 99,271 & 50.96 & 2887 & 133,190 & 21.68 & $0.45(0.43-0.47)^{b}$ & $0.42(0.41-0.44)^{b}$ \\
\hline$\geq 60$ & 2197 & 19,814 & 110.88 & 1131 & 24,044 & 47.04 & $0.48(0.45-0.52)^{b}$ & $0.47(0.43-0.5)^{b}$ \\
\hline \multicolumn{9}{|c|}{ Baseline comorbidities } \\
\hline \multicolumn{9}{|c|}{ Diabetes mellitus } \\
\hline No & 6227 & 193,111 & 32.25 & 3301 & 225,893 & 14.61 & $0.47(0.45-0.5)^{\mathrm{b}}$ & $0.44(0.42-0.46)^{\mathrm{b}}$ \\
\hline Yes & 1906 & 18,916 & 100.76 & 1088 & 25,818 & 42.14 & $0.46(0.42-0.49)^{b}$ & $0.44(0.41-0.47)^{\mathrm{b}}$ \\
\hline \multicolumn{9}{|c|}{ Hypertension } \\
\hline No & 4148 & 174,292 & 23.80 & 2186 & 199,801 & 10.94 & $0.48(0.45-0.5)^{b}$ & $0.43(0.41-0.46)^{b}$ \\
\hline Yes & 3985 & 37,735 & 105.61 & 2203 & 51,910 & 42.44 & $0.44(0.42-0.47)^{b}$ & $0.44(0.42-0.46)^{b}$ \\
\hline \multicolumn{9}{|c|}{ Hyperlipidemia } \\
\hline No & 5328 & 180,288 & 29.55 & 2742 & 210,271 & 13.04 & $0.46(0.44-0.48)^{b}$ & $0.43(0.41-0.45)^{b}$ \\
\hline Yes & 2805 & 31,738 & 88.38 & 1647 & 41,440 & 39.74 & $0.49(0.46-0.52)^{b}$ & $0.46(0.43-0.49)^{b}$ \\
\hline \multicolumn{9}{|c|}{ Congestive heart failure } \\
\hline No & 7942 & 210,670 & 37.70 & 4282 & 249,749 & 17.15 & $0.48(0.46-0.5)^{b}$ & $0.44(0.42-0.45)^{\mathrm{b}}$ \\
\hline Yes & 191 & 1356 & 140.83 & 107 & 1963 & 54.52 & $0.45(0.35-0.57)^{b}$ & $0.46(0.36-0.59)^{b}$ \\
\hline \multicolumn{9}{|c|}{ Cerebrovascular diseases } \\
\hline No & 6867 & 199,807 & 34.37 & 3689 & 235,364 & 15.67 & $0.48(0.46-0.5)^{b}$ & $0.44(0.42-0.45)^{\mathrm{b}}$ \\
\hline Yes & 1266 & 12,220 & 103.60 & 700 & 16,348 & 42.82 & $0.46(0.42-0.5)^{b}$ & $0.45(0.41-0.49)^{b}$ \\
\hline \multicolumn{9}{|c|}{ Depression } \\
\hline No & 7628 & 203,237 & 37.53 & 4091 & 240,799 & 16.99 & $0.48(0.46-0.49)^{b}$ & $0.44(0.42-0.45)^{b}$ \\
\hline Yes & 505 & 8790 & 57.45 & 298 & 10,912 & 27.31 & $0.51(0.44-0.59)^{b}$ & $0.46(0.4-0.53)^{b}$ \\
\hline \multicolumn{9}{|l|}{ Anxiety } \\
\hline No & 6860 & 191,626 & 35.80 & 3702 & 225,730 & 16.40 & $0.48(0.46-0.5)^{b}$ & $0.44(0.42-0.46)^{b}$ \\
\hline Yes & 1273 & 20,401 & 62.40 & 687 & 25,982 & 26.44 & $0.46(0.42-0.5)^{b}$ & $0.43(0.39-0.47)^{\mathrm{b}}$ \\
\hline \multicolumn{9}{|c|}{ Alcoholism or alcohol-related disorders } \\
\hline No & 8093 & 211,137 & 38.33 & 4372 & 250,521 & 17.45 & $0.48(0.46-0.5)^{b}$ & $0.44(0.42-0.46)^{b}$ \\
\hline Yes & 40 & 889 & 44.99 & 17 & 1190 & 14.28 & $0.35(0.2-0.61)^{b}$ & $0.33(0.19-0.6)^{b}$ \\
\hline \multicolumn{9}{|c|}{ Tobacco dependence } \\
\hline No & 8109 & 211,341 & 38.37 & 4374 & 251,047 & 17.42 & $0.48(0.46-0.5)^{b}$ & $0.44(0.42-0.45)^{b}$ \\
\hline Yes & 24 & 685 & 35.02 & 15 & 664 & 22.58 & $0.68(0.35-1.29)$ & $0.47(0.23-0.94)^{c}$ \\
\hline \multicolumn{9}{|l|}{ Obesity } \\
\hline No & 8062 & 210,777 & 38.25 & 4351 & 250,115 & 17.40 & $0.48(0.46-0.5)^{b}$ & $0.44(0.42-0.46)^{b}$ \\
\hline Yes & 71 & 1250 & 56.80 & 38 & 1596 & 23.81 & $0.45(0.3-0.66)^{b}$ & $0.36(0.24-0.55)^{b}$ \\
\hline \multicolumn{9}{|c|}{ Drugs used } \\
\hline \multicolumn{9}{|c|}{ Oral steroids } \\
\hline No & 3458 & 56,042 & 61.70 & 1574 & 74,258 & 21.20 & $0.39(0.37-0.41)^{b}$ & $0.39(0.37-0.42)^{b}$ \\
\hline
\end{tabular}


Table 3 Incidence rates and hazard ratios for coronary heart disease among patients with fibromyalgia with or without acupuncture treatment (Continued)

\begin{tabular}{cclllllll}
\hline Yes & 4675 & 155,985 & 29.97 & 2815 & 177,453 & 15.86 & $0.54(0.52-0.57)^{\mathrm{b}}$ & $0.49(0.46-0.51)^{\mathrm{b}}$ \\
NSAIDs & & & & & & & & \\
No & 543 & 1878 & 289.11 & 175 & 3206 & 54.58 & $0.27(0.23-0.32)^{\mathrm{b}}$ & $0.31(0.26-0.37)^{\mathrm{b}}$ \\
Yes & 7590 & 210,148 & 36.12 & 4214 & 248,505 & 16.96 & $0.49(0.47-0.51)^{\mathrm{b}}$ & $0.45(0.44-0.47)^{\mathrm{b}}$ \\
Statins & & & & & & & & \\
No & 6799 & 179,722 & 37.83 & 3505 & 215,745 & 16.25 & $0.46(0.44-0.48)^{\mathrm{b}}$ & $0.42(0.4-0.44)^{\mathrm{b}}$ \\
Yes & 1334 & 32,305 & 41.29 & 884 & 35,967 & 24.58 & $0.6(0.55-0.66)^{\mathrm{b}}$ & $0.56(0.52-0.61)^{\mathrm{b}}$ \\
\hline
\end{tabular}

IR Incidence rate per 1000 person-years; NSAIDs Nonsteroidal anti-inflammatory drugs

${ }^{\text {a }}$ Adjusted HR adjusted for accepted acupuncture, age, sex, diabetes mellitus, hypertension, hyperlipidemia, congestive heart failure, cerebral vascular diseases, depression, anxiety, alcoholism or alcohol-related disorders, tobacco dependence, obesity, oral steroids, NSAIDs, and statins in Cox proportional hazards regression ${ }^{\mathrm{b}} p<0.001$

c $p<0.05$

cardiac injury [45]. Acupuncture also decreased myocardial infarct areas and preserved cardiac function through heat shock protein 20 (HSP20) and HSP27 in an animal study [27]. These reports demonstrated that acupuncture can protect the heart from ischemia. Future mechanistic study in this area is warranted.

Several limitations in our study are noted. First, the NHIRD did not provide the severity of fibromyalgia, including pain scores, the severity of inflammation, or daily life disabilities. To minimize the confounding factors, we performed a 1:1 propensity score match and found that the baseline characteristics of both cohorts were similar. The percentages of patients who used NSAIDs and oral steroids were also similar in both cohorts. The second limitation is that the NHIRD did not provide data about patients' lifestyles, such as smoking, alcohol drinking, body mass index (BMI), stress, and exercise. Although detailed information

Table 4 Top ten disease categories as reasons for clinical acupuncture visits in the acupuncture cohort

\begin{tabular}{ll}
\hline Disease categories (ICD-9-CM codes) & $\begin{array}{l}\text { Acupuncture cohort } \\
(n=58,899)\end{array}$ \\
\hline $\begin{array}{l}\text { Disorders of musculoskeletal system and } \\
\text { connective tissue (710-739) }\end{array}$ & 42,488 \\
$\begin{array}{l}\text { Injury and poisoning (800-999) } \\
\text { Symptoms, signs, and ill-defined conditions }\end{array}$ & 36,465 \\
$\begin{array}{l}\text { (780-799) } \\
\text { Disorders of nervous system (320-389) }\end{array}$ & 2802 \\
Disorders of digestive system (520-579) & 1714 \\
Disorders of respiratory system (460-519) & 1497 \\
Disorders of circulatory system (390-459) & 850 \\
Disorders of genitourinary system (580-629) & 725 \\
Disorders of skin and subcutaneous tissue (680- & 335 \\
709) & \\
Mental disorders (290-319) & 283 \\
\hline
\end{tabular}

ICD-9-CM International Classification of Diseases, Ninth Revision, Clinical Modification

More than one ICD-9-CM code can be recorded when patients receive acupuncture about smoking exposure, alcohol drinking, and BMI was not available, we were able to acquire information on illnesses resulting from these personal habits and lifestyles. We conducted a 1:1 propensity score match that included the diagnoses of alcoholism or alcohol-related disorders, tobacco dependence, and obesity. Patients with these disorders were similar in both cohorts, and the protective effect of acupuncture on CHD was significant in patients with fibromyalgia with these comorbid diseases. However, there was still an underestimation of smoking exposure and alcohol drinking in both cohorts. On the basis of our findings in this matched cohort study, we have designed a prospective participant- and assessor-blinded, randomized controlled trial to investigate the efficacy and mechanisms of acupuncture in patients with fibromyalgia (ClinicalTrials.gov identifier: NCT02583334). These lifestyle factors are recorded using a patient-reported questionnaire and should be able to provide more information regarding lifestyle. The third limitation was that detailed information on selected acupoints was not provided in the dataset. Acupoint selection was individualized according to the TCM diagnosis of patients and the experience of doctors, which is more like an effectiveness study. Most studies on the efficacy of acupuncture fix the acupoints to standardize their treatment protocol. The clinical trial of acupuncture in patients with fibromyalgia we conducted also fixed the acupoints as LI4, LI11, LR3, ST36, SP6, and GB34, which are commonly used acupoints for the treatment of patients with fibromyalgia.

\section{Conclusions}

Our present study confirms that acupuncture reduced the risk of CHD in patients with fibromyalgia in Taiwan. This noteworthy finding can provide some hints for further clinical and mechanistic studies.

\section{Abbreviations}

BMI: Body mass index; CHD: Coronary heart disease; DM: Diabetes mellitus; FDA: U.S. Food and Drug Administration; HSP: Heat shock protein; ICD-9CM: International Classification of Diseases, Ninth Revision, Clinical 
Modification; IR: Incidence rate; LHID 2000: Longitudinal Health Insurance Database 2000; NHI: National Health Insurance; NHIA: National Health Insurance Administration; NHIRD: National Health Insurance Research Database; NMDAR: N-methyl-D-aspartate receptor; NSAID: Nonsteroidal anti-inflammatory drug; QOL: Quality of life; TCM: Traditional Chinese medicine; TRPV1: Transient receptor potential vanilloid 1

\section{Acknowledgements}

This study was supported in part by China Medical University under the Aim for Top University Plan of the Ministry of Education, Taiwan. It was also supported by the Taiwan Ministry of Health and Welfare Clinical Trial and Research Center of Excellence (MOHW105-TDU-B-212-133019). This study was based in part on data derived from the NHIRD, provided by the NHIA, and the Ministry of Health and Welfare, and managed by the National Health Research Institutes. The interpretations and conclusions reported do not represent those of the NHIA, the Ministry of Health and Welfare, or the National Health Research Institutes. The funding agencies had no role in the study design, data collection and analyses, results interpretation, or publication of this study.

\section{Funding}

This study was supported in part by China Medical University under the Aim for Top University Plan of the Ministry of Education, Taiwan. It was also supported by the Taiwan Ministry of Health and Welfare Clinical Trial and Research Center of Excellence (MOHW105-TDU-B-212-133019).

\section{Availability of supporting data}

All data of this study are deposited in a properly managed public repository. We examined and analyzed datasets released from the Taiwan NHIRD (http://nhird.nhri.org.tw/en/index.html), maintained and managed by National Health Research Institutes (http://www.nhri.org.tw/), Taiwan. The use of the datasets is limited to research purposes only. Applicants must follow the Computer-Processed Personal Data Protection Law (http://www.winklerpartners.com/?p=987) and related regulations of the National Health Insurance Administration and the National Health Research Institutes, and the agreement must be signed by the applicant and his/her supervisor upon application submission. All applications are reviewed for approval of data release.

\section{Authors' contributions}

MYW contributed to the conception and design of the study, participated in the interpretation of clinical data, and drafted the manuscript. $\mathrm{MCH}$ participated in the interpretation of clinical data and acupuncture treatment approaches. JHC performed the statistical analysis. MFS participated in the interpretation of clinical data. YCL participated in the interpretation of clinical data and acupuncture treatment approaches and also helped to finalize the manuscript. HRY conceived of the study, contributed to the interpretation of clinical data, and finalized the manuscript. All authors read and approved the final manuscript.

\section{Competing interests}

The authors declare that they have no competing interests.

\section{Consent for publication}

Not applicable in this section.

\section{Ethics approval and consent to participate}

This study was approved by the research ethics committee of China Medical University and Hospital (CMUH104-REC2-115) and by the National Health Research Institute, the data holder of the $\mathrm{NHI}$ database. The need for patient consent was exempted on the basis of the total anonymity of all research data in this study.

\section{Author details}

${ }^{1}$ Research Center for Traditional Chinese Medicine, Department of Medical Research, China Medical University Hospital, 2 Yude Road, North District, Taichung, Taiwan. ${ }^{2}$ Department of Chinese Medicine, China Medical University Hospital, 2 Yude Road, North District, Taichung, Taiwan. ${ }^{3}$ Graduate Institute of Chinese Medicine, School of Chinese Medicine, College of Chinese Medicine, China Medical University, Taichung, Taiwan. ${ }^{4}$ Health Data Management Office, China Medical University Hospital, 2 Yude Road, North
District, Taichung, Taiwan. ${ }^{5}$ Research Center for Chinese Medicine \& Acupuncture, China Medical University, Taichung, Taiwan. ${ }^{6}$ Graduate Institute of Acupuncture Science, College of Chinese Medicine, China Medical University, Taichung, Taiwan.

Received: 19 October 2016 Accepted: 19 January 2017

Published online: 28 February 2017

\section{References}

1. Chinn S, Caldwell W, Gritsenko K. Fibromyalgia pathogenesis and treatment options update. Curr Pain Headache Rep. 2016;20:25.

2. Arnold LM, Gebke KB, Choy EH. Fibromyalgia: management strategies for primary care providers. Int J Clin Pract. 2016;70:99-112

3. Wolfe F, Clauw DJ, Fitzcharles MA, Goldenberg DL, Katz RS, Mease P, et al. The American College of Rheumatology preliminary diagnostic criteria for fibromyalgia and measurement of symptom severity. Arthritis Care Res (Hoboken). 2010;62:600-10.

4. Queiroz LP. Worldwide epidemiology of fibromyalgia. Curr Pain Headache Rep. 2013;17:356.

5. Weir PT, Harlan GA, Nkoy FL, Jones SS, Hegmann KT, Gren LH, et al. The incidence of fibromyalgia and its associated comorbidities: a populationbased retrospective cohort study based on International Classification of Diseases, 9th Revision codes. J Clin Rheumatol. 2006;12:124-8.

6. Walitt B, Nahin RL, Katz RS, Bergman MJ, Wolfe F. The prevalence and characteristics of fibromyalgia in the 2012 National Health Interview Survey. PLoS One. 2015;10, e0138024.

7. Cho Kl, Lee JH, Lee HG, Kim SM, Kim TI. Assessment of myocardial function in patients with fibromyalgia and the relationship to chronic emotional and physical stress. Korean Circ J. 2010;40:74-80.

8. Su CH, Chen JH, Lan JL, Wang YC, Tseng CH, Hsu CY, et al. Increased risk of coronary heart disease in patients with primary fibromyalgia and those with concomitant comorbidity - a Taiwanese population-based cohort study. PLoS One. 2015;10, e0137137.

9. Blumenthal DE, Malemud CJ. Recent strategies for drug development in fibromyalgia syndrome. Expert Rev Neurother. 2016;16:1407-11.

10. Langhorst J, Klose P, Musial F, Irnich D, Häuser W. Efficacy of acupuncture in fibromyalgia syndrome-a systematic review with a meta-analysis of controlled clinical trials. Rheumatology (Oxford). 2010;49:778-88.

11. Lin JG, Hsieh CL, Lin YW. Analgesic effect of electroacupuncture in a mouse fibromyalgia model: roles of TRPV1, TRPV4, and pERK. PLoS One. 2015;10, e0128037.

12. Hsing AW, loannidis JP. Nationwide population science: lessons from the Taiwan National Health Insurance Research Database. JAMA Intern Med. 2015;175:1527-9.

13. Chang CC, Lee YC, Lin CC, Chang CH, Chiu CD, Chou LW, et al. Characteristics of traditional Chinese medicine usage in patients with stroke in Taiwan: a nationwide population-based study. J Ethnopharmacol. 2016; 186:311-21.

14. Yen HR, Lai WY, Muo CH, Sun MF. Characteristics of traditional Chinese medicine use in pediatric cancer patients: a nationwide, retrospective, Taiwanese-registry, population-based study. Integr Cancer Ther. doi:10.1177/ 1534735416659357.

15. Lien AS, Jiang YD, Mou CH, Sun MF, Gau BS, Yen HR. Integrative traditional Chinese medicine therapy reduces the risk of diabetic ketoacidosis in patients with type 1 diabetes mellitus. J Ethnopharmacol. 2016;191:324-30.

16. Liao HH, Yeh CC, Lin CC, Chen BC, Yeh MH, Chang KM, et al. Prescription patterns of Chinese herbal products for patients with fractures in Taiwan: a nationwide population-based study. J Ethnopharmacol. 2015;173:11-9.

17. Shih CC, Liao CC, Sun MF, Sun YC, Wen CP, Morisky DE, et al. Retrospective cohort study comparing stroke recurrence rate in ischemic stroke patients with and without acupuncture treatment. Medicine (Baltimore). 2015;94:e1572.

18. LaRosa JC, Pedersen TR, Somaratne R, Wasserman SM. Safety and effect of very low levels of low-density lipoprotein cholesterol on cardiovascular events. Am J Cardiol. 2013;111:1221-9.

19. Chen FP, Kung YY, Chen TJ, Hwang SJ. Demographics and patterns of acupuncture use in the Chinese population: the Taiwan experience. J Altern Complement Med. 2006:12:379-87.

20. Cramer H, Chung VC, Lauche R, Zhang Y, Zhang A, Langhorst J, et al. Characteristics of acupuncture users among internal medicine patients in Germany. Complement Ther Med. 2015;23:423-9. 
21. Vas J, Santos-Rey K, Navarro-Pablo R, Modesto M, Aguilar I, Campos MÁ, et al. Acupuncture for fibromyalgia in primary care: a randomised controlled trial. Acupunct Med. 2016;34:257-66.

22. Hadianfard MJ, Hosseinzadeh PM. A randomized clinical trial of fibromyalgia treatment with acupuncture compared with fluoxetine. Iran Red Crescent Med J. 2012:14:631-40.

23. Martin DP, Sletten CD, Williams BA, Berger $\mathbb{H}$. Improvement in fibromyalgia symptoms with acupuncture: results of a randomized controlled trial. Mayo Clin Proc. 2006;81:749-57.

24. Branco JC, Bannwarth B, Failde I, Abello Carbonell J, Blotman F, Spaeth M, et al. Prevalence of fibromyalgia: a survey in five European countries. Semin Arthritis Rheum. 2010;39:448-53.

25. Crofford $\sqcup$, Rowbotham MC, Mease PJ, Russell IJ, Dworkin RH, Corbin AE, et al. Pregabalin for the treatment of fibromyalgia syndrome: results of a randomized, double-blind, placebo-controlled trial. Arthritis Rheum. 2005;52:1264-73.

26. Ho JM, Tricco AC, Perrier L, Chen M, Juurlink DN, Straus SE. Risk of heart failure and edema associated with the use of pregabalin: a systematic review. Syst Rev. 2013;2:25.

27. Lee SMK, Yoon KH, Park J, Kim HS, Woo JS, Lee SR, et al. Additional effects of back-shu electroacupuncture and moxibustion in cardioprotection of rat ischemia-reperfusion injury. Evid Based Complement Alternat Med. 2015; 2015:625645.

28. Ma L, Cui B, Shao Y, Ni B, Zhang W, Luo Y, et al. Electroacupuncture improves cardiac function and remodeling by inhibition of sympathoexcitation in chronic heart failure rats. Am J Physiol Heart Circ Physiol. 2014;306:H1464-71.

29. Sluka KA, Clauw DJ. Neurobiology of fibromyalgia and chronic widespread pain. Neuroscience. 2016;338:114-29.

30. de Tommaso M, Delussi M, Ricci K, D’Angelo G. Abdominal acupuncture changes cortical responses to nociceptive stimuli in fibromyalgia patients. CNS Neurosci Ther. 2014;20:565-7.

31. Lu KW, Hsieh CL, Yang J, Lin YW. Effects of electroacupuncture in a mouse model of fibromyalgia: role of $\mathrm{N}$-methyl-D-aspartate receptors and related mechanisms. Acupunct Med. doi:10.1136/acupmed-2015-010986.

32. McGee MA, Abdel-Rahman AA. N-methyl-D-aspartate receptor signaling and function in cardiovascular tissues. J Cardiovasc Pharmacol. 2016;68:97-105.

33. Shi S, Liu T, Wang D, Zhang Y, Liang J, Yang B, et al. Activation of N-methylD-aspartate receptors reduces heart rate variability and facilitates atrial fibrillation in rats. Europace. doi:10.1093/europace/euw086.

34. Seo BK, Sung WS, Park YC, Baek YH. The electroacupuncture-induced analgesic effect mediated by $5-\mathrm{HT}_{1}, 5-\mathrm{HT}_{3}$ receptor and muscarinic cholinergic receptors in rat model of collagenase-induced osteoarthritis. BMC Complement Altern Med. 2016;16:212

35. Seidel MF, Weinreich GF, Stratz T, Müller W. 5-HT3 receptor antagonists regulate autonomic cardiac dysfunction in primary fibromyalgia syndrome. Rheumatol Int. 2007;27:1025-30.

36. Xiong S, Wang P, Ma L, Gao P, Gong L, Li L, et al. Ameliorating endothelial mitochondrial dysfunction restores coronary function via transient receptor potential vanilloid 1-mediated protein kinase A/uncoupling protein 2 pathway. Hypertension. 2016;67:451-60.

37. Shergis JL, Ni X, Jackson ML, Zhang AL, Guo X, Li Y, et al. A systematic review of acupuncture for sleep quality in people with insomnia. Complement Ther Med. 2016;26:11-20.

38. Tobaldini E, Costantino G, Solbiati M, Cogliati C, Kara T, Nobili L, et al. Sleep, sleep deprivation, autonomic nervous system and cardiovascular diseases. Neurosci Biobehav Rev. doi:10.1016/j.neubiorev.2016.07.004.

39. Markkula RA, Kalso EA, Kaprio JA. Predictors of fibromyalgia: a populationbased twin cohort study. BMC Musculoskelet Disord. 2016;17:29.

40. Dhar AK, Barton DA. Depression and the link with cardiovascular disease. Front Psychiatry. 2016;7:33

41. Le JJ, Yi T, Qi L, Li J, Shao L, Dong JC. Electroacupuncture regulate hypothalamic-pituitary-adrenal axis and enhance hippocampal serotonin system in a rat model of depression. Neurosci Lett. 2016;615:66-71.

42. Lu J, Shao RH, Hu L, Tu Y, Guo JY. Potential antiinflammatory effects of acupuncture in a chronic stress model of depression in rats. Neurosci Lett. 2016;618:31-8

43. Torres-Rosas R, Yehia G, Peña G, Mishra P, del Rocio Thompson-Bonilla M, Moreno-Eutimio MA, et al. Dopamine mediates vagal modulation of the immune system by electroacupuncture. Nat Med. 2014;20:291-5.

44. Zhao C, Bao C, Li J, Zhu Y, Wang S, Yang L, et al. Moxibustion and acupuncture ameliorate Crohn's disease by regulating the balance between
Th17 and Treg cells in the intestinal mucosa. Evid Based Complement Alternat Med. 2015;2015:938054.

45. Wang Q, Liang D, Wang F, Li W, Han Y, Zhang W, et al. Efficacy of electroacupuncture pretreatment for myocardial injury in patients undergoing percutaneous coronary intervention: a randomized clinical trial with a 2-year follow-up. Int J Cardiol. 2015;194:28-35.

\section{Submit your next manuscript to BioMed Central and we will help you at every step:}

- We accept pre-submission inquiries

- Our selector tool helps you to find the most relevant journal

- We provide round the clock customer support

- Convenient online submission

- Thorough peer review

- Inclusion in PubMed and all major indexing services

- Maximum visibility for your research

Submit your manuscript at www.biomedcentral.com/submit
) Biomed Central 